\section{MÉTODO MÃE-CANGURU: PERCEPÇÃO DA EQUIPE DE ENFERMAGEM NA PROMOÇÃO À SAÚDE DO NEONATO}

\author{
Kangaroo-Mother Method: perception of the nursing staff in \\ the neonate's health promotion
}

\section{Método Madre-Canguru: percepción del equipo de enfermería en la promoción de la salud del neonato}

\section{RESUMO}

Objetivo: Conhecer a percepção da equipe de enfermagem sobre o Método Mãe-Canguru da maternidade de um hospital de ensino. Métodos: Pesquisa qualitativa, realizada entre setembro e outubro de 2010, na Maternidade Maria Barbosa do Hospital Universitário Clemente de Faria, Montes Claros-MG, Brasil, com cinco profissionais de enfermagem. Utilizaram-se entrevistas não diretivas para coleta dos dados, os quais foram analisados por meio da análise de conteúdo, emergindo duas categorias de análise: "Percebendo o Método Mãe-Canguru como um estímulo de ligação entre a mãe e o filho" e "Compreendendo o Método Mãe-Canguru como benefício para o desenvolvimento do recém-nascido". Resultados: Os profissionais enxergam o Método Mãe-Canguru como estímulo para o estabelecimento do vínculo e apego mãe-filho, permitindo que esse binômio mantenha a união que foi construída desde a vida intrauterina. O Método Mãe-Canguru é considerado um fator relevante na recuperação do recém-nascido, pois propicia aumento de peso, estabilidade dos dados vitais e estímulo à amamentação. Destaca-se ainda que, para os entrevistados, a capacitação dos profissionais, por meio da educação continuada, quanto à realização da técnica é essencial para ofertar o cuidado humanizado na assistência neonatal. Conclusão: Os profissionais de enfermagem entrevistados compreendem o Método Mãe-Canguru aplicado na sua assistência diária como uma busca pela humanização do cuidado ao recémnascido, uma forma de estímulo da ligação entre mãe e filho, apresentando-se como fator de relevância na recuperação do recém-nascido e proporcionando melhoria na saúde do neonato.

Descritores: Maternidades; Método Mãe-Canguru; Equipe de Enfermagem.

\section{ABSTRACT}

Objective: To know about the perception of the nursing staff about the Kangaroo-Mother Method in the maternity ward of a teaching hospital. Methods: Qualitative research, conducted in September and October 2010, in Maria Barbosa Maternity of Clemente de Faria University Hospital, Montes Claros, Minas Gerais, Brazil, with five nursing professionals. Non-directive interviews were used for data collection, which were analyzed by means of the content analysis, emerging two categories of analysis: 'Realizing the Kangaroo-Mother Method as a stimulus to the connection between the mother and the child' and 'Understanding the Kangaroo-Mother Method as a benefit to the newborn's development'. Results: The professionals see the Kangaroo-Mother Method as a stimulus to the establishment of the mother-child bond, allowing this binomial to keep the union that was built since the intrauterine life. The Kangaroo-Mother Method is regarded as a relevant factor in the newborn's recovery, given that it encourages weight gain, stability of vital data and stimulus to breastfeeding. It is also noticeable that, for the interviewees, professional training through continuing education on the performance of the technique is essential for providing humanized care in neonatal assistance. Conclusion: The nursing professionals interviewed understand the Kangaroo-Mother Method applied in their daily assistance as a search for humanization of care to the newborn, as a form of stimulation of the connection between mother and son, being presented as a relevant factor in the newborn's recovery, providing improvement in the health of the neonate.

Descriptors: Maternity; Kangaroo-Mother Method; Nursing, Team.
Artigo Original

Luís Paulo Souza e Souza ${ }^{(1)}$

Elza Vasconcelos de Souza ${ }^{(2)}$

Gisele Cristina Soares Gomes ${ }^{(2)}$

Daniella Fagundes Souto(1)

Luciana Barbosa Pereira ${ }^{(1)}$

Maria Ângela Martins Pinheiro(3)

Carla Silvana de Oliveira e

Silva(2)

Clara de Cássia Versiani ${ }^{(1)}$

1) Universidade Estadual de Montes Claros - UNIMONTES - Montes Claros (MG) -

Brasil

2) Faculdades de Saúde e Desenvolvimento Humano Santo Agostinho - FASADHU Montes Claros (MG) - Brasil

3) Faculdades Integradas do Norte de Minas - FUNORTE - Montes Claros (MG) - Brasil
Recebido em: 22/11/2013

Revisado em: 05/01/2014 Aceito em: 18/03/2014 


\section{RESUMEN}

Objetivo: Conocer la percepción del equipo de enfermería sobre el Método Madre-Canguru de la maternidad de un hospital universitario. Métodos: Investigación cualitativa realizada en septiembre y octubre de 2010 en la maternidad María Barbosa del Hospital Universitario Clemente de Faria, Montes Claros-MG, Brasil, con cinco profesionales de la enfermería. Para la recogida de datos se utilizó entrevistas no directivas las cuales fueron analizadas a través del análisis de contenido, identificándose dos categorías de análisis: "La percepción del Método MadreCanguru como un estímulo de relación entre madre e hijo" y "La comprensión del Método Madre-Canguru como beneficio para el desarrollo del recién-nacido". Resultados: Los profesionales identifican el Método Madre-Canguru como estímulo para el establecimiento de la relación y apego madre-hijo, permitiendo que ese binomio mantenga la unión que fue construida en la vida intra-uterina. El Método Madre-Canguru es considerado un factor relevante en la recuperación del recién-nacido porque conlleva al aumento de peso, estabilidad de datos vitales y estímulo al amamantamiento. Se destaca, todavía, que para los entrevistados, la capacitación de los profesionales sobre la realización de la técnica a través de la educación continuada es fundamental para ofrecer el cuidado humanizado en la asistencia neonatal. Conclusión: Los profesionales de enfermería entrevistados comprenden que el Método Madre-Canguru aplicado en la asistencia a diario como una búsqueda de la humanización del cuidado al recién-nacido, una forma de estímulo de la relación madre-hijo presentándose como factor relevante en la recuperación del recién-nacido y promoviendo la mejoría de su salud.

Descriptores: Maternidades; Método Madre-Canguru; Grupo de Enfermería.

\section{INTRODUÇÃO}

O Método Mãe-Canguru (MMC) é uma tecnologia de assistência neonatal que consiste em manter o bebê na posição vertical, junto ao peito de um adulto. Foi criado em 1979, pelo Doutor Edgar Rey Sanabria, no Instituto Materno-Infantil de Bogotá, na Colômbia. O método consistia em posicionar o recém-nascido $(\mathrm{RN})$ prematuro entre os seios maternos, em contato pele a pele, na posição supina. Dessa forma, mantendo-se aquecido com o calor do corpo de sua mãe, o RN poderia sair mais cedo da incubadora e, consequentemente, ter alta, minimizando dois graves problemas da época: superlotação e infecção ${ }^{(1)}$.

No Brasil, as instituições pioneiras que implantaram o MMC foram o Hospital Guilherme Álvaro, localizado em Santos, no interior de São Paulo, em 1991, e o Instituto Materno-Infantil de Pernambuco, em Recife, em 1993. Foi através da Norma de Atenção Humanizada ao RecémNascido de Baixo Peso (RNBP) que, a partir de dezembro de 1999, a área da Criança do Ministério da Saúde (MS) veio adotar o MMC como uma Política Nacional de Saúde, inserida no contexto da humanização da assistência neonatal ${ }^{(2)}$, por meio do Projeto Mãe-Canguru, Portaria $n^{\circ}$ $693 \mathrm{GM} / \mathrm{MS}^{(3)}$.

O MMC é realizado em três etapas. Na primeira, o $\mathrm{RN}$ prematuro é internado na unidade intensiva e a mãe e a família recebem informações sobre as condições de saúde, os procedimentos hospitalares, a amamentação e os cuidados que devem realizar, a fim de melhorar a compreensão da prematuridade. Na segunda etapa, já com o ganho de peso e a estabilização do RN, o acompanhamento da mãe pode ser contínuo e a posição canguru já é possível, podendo ser utilizada pelo tempo em que for prazerosa para ambos. A decisão pela participação no método deve resultar de um consenso entre mãe, família e equipe do hospital. A mãe aprende a identificar as alterações que podem ocorrer com a criança, como pausas respiratórias, mudança de coloração da pele, entre outras. A terceira etapa se finaliza com a alta hospitalar e, nesse processo, a mãe deve estar preparada, assim como os familiares, pois é indispensável o comprometimento com a continuidade do método. O RN deve pesar no mínimo 1.500 gramas e ter a capacidade de sucção exclusiva ao peito. $\mathrm{O}$ acompanhamento nessa fase é ambulatorial e sua função se caracteriza pela realização de exames físicos para a verificação do desenvolvimento da criança, além de orientação e acompanhamento em possíveis consultas e tratamento especializados ${ }^{(4)}$.

Desta forma, o MMC abrange questões como os cuidados técnicos com o bebê prematuro ou de baixo peso (manuseio, atenção às necessidades individuais, cuidados com luz, som, dor); acolhimento à família; promoção do vínculo mãe/bebê e do aleitamento materno; $\mathrm{e}$ acompanhamento ambulatorial após a alta ${ }^{(1,5)}$.

Dados da pesquisa "Prematuridade e suas possíveis causas" mostram que, em 2011, no Brasil, a prevalência de crianças prematuras foi de $11,8 \%$, tendo as regiões Sudeste e Sul as maiores taxas, totalizando $12,5 \%$ e $12 \%$, respectivamente, seguidas pelas regiões Centro-Oeste $(11,5 \%)$, Nordeste $(10,9 \%)$ e Norte $(10,8 \%)$. Os estados que lideram o ranking são Minas Gerais (12,7\%) e o Distrito Federal (13\%). A alta prevalência de prematuridade tem importantes repercussões sociais e econômicas, com a demanda crescente de Unidades de Terapia Intensivas Neonatal ${ }^{(6)}$. Diante disso, destaca-se a importância e eficiência do MMC na busca pela qualidade da assistência prestada aos RN prematuros, uma vez que proporciona um cuidado individualizado, aliado à tecnologia e à sensibilidade, pois se acredita que favorece a entrada dos pais na unidade neonatal, promovendo o acolhimento da família, ampliando o foco do cuidado para além do $\mathrm{RN}$ prematuro $^{(7)}$ e proporcionando, ainda, melhor qualidade de 
vida, redução dos índices de mortalidade nesse segmento, implantação e manutenção de baixo custo na saúde ${ }^{(8)}$.

A participação de enfermeiros em grupos de discussão e na aplicabilidade global do método vem sendo cada vez mais prestigiada, fornecendo contribuições para o sucesso da implementação do $\mathrm{MMC}^{(9)}$.

$\mathrm{O}$ interesse pelo tema surgiu a partir das experiências vivenciadas por parte dos autores, pois são profissionais que lidaram ou lidam direta ou indiretamente com a assistência a RN prematuros, utilizando a Metodologia Canguru. Com o passar do tempo, inquietações foram surgindo à medida que os profissionais se apresentavam comprometidos com a qualidade da assistência prestada e observavam que as ações, algumas vezes, eram realizadas de maneira rotineira e pouco refletidas. Com isso, surge a seguinte indagação ou questão norteadora: "Como os membros da equipe de enfermagem compreendem o Método Mãe-Canguru na assistência neonatal?".

Este trabalho se justifica uma vez que é importante conhecer os significados que a equipe de enfermagem apresenta em relação ao $\mathrm{MMC}$, já que, com a introdução desse método, a equipe foi compelida a reorganizar sua prática assistencial, tendo que rever comportamentos e atitudes por meio de mudanças impostas, não havendo espaço para que pudesse transformar as ações de maneira consciente $^{(7)}$. Muito se fala, inclusive pelo MS, na necessidade de capacitar as equipes, mas não se fala da real importância de analisar e refletir sobre a compreensão que a equipe de enfermagem apresenta em relação ao método, considerando as possibilidades de aplicação e reflexões sobre os benefícios e implicações que o ele acarreta aos $\mathrm{RN}^{(6)}$.

A partir do exposto, o presente estudo objetivou conhecer a percepção da equipe de enfermagem sobre o Método Mãe-Canguru da maternidade de um hospital de ensino.

\section{MÉTODOS}

Trata-se de uma pesquisa descritiva, com abordagem qualitativa $^{(10-11)}$, realizada na Maternidade Maria Barbosa do Hospital Universitário Clemente de Faria, em Montes Claros-MG, Brasil.

A Maternidade Maria Barbosa é composta por 30 leitos e registra uma média de 180 nascimentos por mês. Apresenta um percurso significativo no processo de humanização da assistência materno-infantil da cidade de Montes Claros e diversas cidades do norte de Minas Gerais e sul da Bahia, sendo referência regional para atendimento às gestantes de alto risco, soropositivas e portadoras do Vírus da Imunodeficiência Humana (HIV). Nessa maternidade, adota-se a posição canguru num espaço denominado "hotelzinho", composto de seis leitos, onde mãe e filho permanecem juntos até que o $\mathrm{RN}$ atinja $2 \mathrm{~kg}$ e esteja em boas condições clínicas para alta.

Fizeram parte do estudo profissionais da equipe de enfermagem da referida maternidade, os quais atenderam aos seguintes critérios: ser profissional da equipe de enfermagem (auxiliar de enfermagem, técnico de enfermagem ou enfermeiro) e ser profissional atuante na maternidade há mais de seis meses, pois seria o tempo necessário para que o profissional atuasse em algum momento como facilitador do processo de humanização ao $\mathrm{RN}$ por meio do $\mathrm{MMC}$. Todos os profissionais de enfermagem da maternidade foram comunicados previamente sobre a pesquisa, sendo esclarecidos sobre os objetivos e a importância do estudo. Aqueles que aceitaram participar do estudo e se encaixaram nos critérios de inclusão estabelecidos foram convidados a agendar a entrevista para o dia em que estivessem de plantão.

A coleta de dados ocorreu nos meses de setembro e outubro de 2010, por meio de entrevistas não diretivas, gravadas por dois dos autores, na própria maternidade, onde os profissionais foram abordados nos dias agendados e em seus horários de descanso, para que a rotina do serviço não fosse afetada, tendo sido aplicada em local tranquilo, a fim de evitar interrupções. As questões norteadoras da entrevista foram: "Conte para mim sobre o significado do MMC para você enquanto profissional de enfermagem" e "Fale para mim sobre a importância desse método para a assistência de enfermagem". Ressalta-se que o número de participantes não foi predeterminado, pois, na pesquisa qualitativa, o foco não é obter respostas quantificáveis, mas abranger a totalidade do problema investigado em suas múltiplas dimensões. Dessa forma, houve a saturação com cinco entrevistadas, findando-se a coleta, tendo em vista a reincidência dos dados ${ }^{(10)}$. Participaram deste estudo quatro técnicas de enfermagem e uma enfermeira, as quais apresentavam idade entre 28 e 42 anos.

Para organizar os resultados, as entrevistas foram transcritas na íntegra. Na condução da análise dos dados, foi utilizada a técnica de análise do conteúdo tipo categorial ${ }^{(12)}$, a qual apresenta os seguintes passos: pré-análise (organização do corpus de conhecimento para análise); leitura flutuante; leitura exaustiva; escolha e codificação das unidades de análise; e elaboração das categorias ("Percebendo o Método Mãe-Canguru como um estímulo de ligação entre a mãe e o filho" e "Compreendendo o Método Mãe-Canguru como benefício para o desenvolvimento do recém-nascido"), buscando-se as relações entre elas. Para manter o anonimato dos entrevistados, foram utilizados pseudônimos com nomes de mulheres. 
O estudo foi aprovado pela Comissão de Ética do Hospital Universitário Clemente de Faria, a qual autorizou, para fins acadêmicos, a divulgação dos dados. Ressalta-se, ainda, que foi aprovado pelo Comitê de Ética em Pesquisa da Universidade Estadual de Montes Claros, por meio do parecer $n^{\circ} 2155$. Todas as entrevistadas assinaram o Termo de Consentimento Livre e Esclarecido.

\section{RESULTADOS E DISCUSSÃO}

Neste espaço, serão apresentadas as categorias temáticas que emergiram do estudo.

\section{Percebendo o Método Mãe-Canguru como um estímulo de ligação entre a mãe e o filho}

A categoria "Percebendo o Método Mãe-Canguru como um estímulo de ligação entre a mãe e o filho" ressalta a humanização da assistência. Está fundamentada na reaproximação dos pais com seus filhos nas unidades de internamento hospitalar ${ }^{(13)}$, promovendo uma vivência única, deixando as mães mais próximas de seus bebês de um jeito semelhante ao intraútero ${ }^{(14)}$. Dentro da ação de implementação do MMC no Brasil, a enfermagem tem contribuído para a eficácia desse programa por meio de uma assistência pautada na dedicação e humanização do cuidado, proporcionando maior interação entre família, bebê prematuro e equipe de saúde ${ }^{(13)}$.

A equipe de enfermagem compreende o MMC como um estímulo de ligação entre a mãe e o filho, permitindo que esse binômio mantenha a união que foi construída desde a vida intrauterina, ao favorecer uma maior aproximação entre eles, podendo ser evidenciado nas falas a seguir:

"Aumenta o vínculo entre mãe e filho." (Maria)

"A gente vê [...] que o bebê que fica no Método Mãe Canguru fica muito mais apegado à mãe." (Francisca)

"Esse método estimula a amamentação." (Kátia)

"Quando a mãe aceita fazer o Método Canguru, a gente vê [...] que ela fica feliz." (Sheila)

Pelas falas, percebe-se que os sujeitos visualizaram o MMC como favorecedor para o estabelecimento do vínculo e apego mãe-filho. Abordaram a importância dessa aproximação para o estímulo da amamentação e entenderam que o método leva a uma satisfação pessoal manifestada pela assistência que foi orientada e estimulada.

As vivências das mães com MMC estão relacionadas ao aumento do vínculo mãe-bebê e à diminuição do tempo de separação do RN da família, melhorando o relacionamento da mãe com a família, entre eles e com a equipe ${ }^{(13,15)}$.

Ressalta-se que um dos principais objetivos do MMC é o estímulo ao aleitamento materno ${ }^{(13)}$. Estudos revelaram que a prática do MMC favorece o aleitamento materno devido a um maior contato entre mãe e filho, além do posicionamento facilitado por esse método. Consequentemente, proporciona aleitamento materno exclusivo mesmo após a alta hospitalar ${ }^{(14,16)}$.

Assim, com o estímulo desse vínculo, o MMC também permite o cuidado materno para com o seu RN. Portanto, ao visualizarem o MMC como assistência, os profissionais de enfermagem foram percebendo que esse cuidado deve ser valorizado e estimulado, levando uma maior tranquilidade e, consequentemente, à segurança da equipe com a mãe.

\begin{abstract}
"O cuidado que a gente ensina e orienta a terem aqui é o mesmo cuidado que elas devem ter em casa." (Maria)

"A gente deixa, por exemplo, uma hora... Mas muitas vezes a mãe deixa bem mais tempo." (Sheila)

"Ao vivenciarmos esse método com a mãe e o bebê, a gente fica mais segura que a maioria das mães está cuidando como deve ou como pelo menos deveria ser." (Maria)
\end{abstract}

O MMC proporciona maior competência e confiança dos pais nos cuidados com seu filho, mesmo antes da alta hospitalar ${ }^{(15)}$. Assim, autores destacam que as mães consideram que o MMC representa uma oportunidade de aprendizagem quanto ao cuidado com seu filho e que o vínculo afetivo é relevante e proporciona a recuperação do bebê ${ }^{(14,17)}$.

Todavia, alguns profissionais entrevistados perceberam que as mães acabam recebendo uma carga de responsabilidade com a realização desse método, podendo acarretar em dificuldades na sua vivência em relação a ele.

\section{"A mãe acaba substituindo em parte esse cuidado." (Maria) \\ "Na verdade, tem mães que ficam mais tempo, tem mães que às vezes não aguentam." (Sheila)}

No MMC, o protagonista é o bebê-prematuro. Observa-se, então, que a enfermagem responsabiliza-se excessivamente pela mulher-mãe, sendo importante que a equipe de saúde atente para as dificuldades com as quais a mãe pode se deparar na vivência do método, amparando-a nos momentos mais frágeis ${ }^{(15,18)}$. É importante destacar que a participação da mãe nesse método não é obrigatória e que, segundo o MS, é sugerido que um adulto realize o posicionamento, entretanto, o cuidado ofertado pela mãe é visto como uma forma particular e sua presença favorece o aleitamento materno, a formação do vínculo e a boa recuperação do $\mathrm{RN}^{(18)}$.

\section{Compreendendo o Método Mãe-Canguru como benefício para o desenvolvimento do recém-nascido}

A categoria "Compreendendo o Método Mãe-Canguru como benefício para o desenvolvimento do recém-nascido" 
foi vista como um diferencial no modelo de humanização na assistência ao RN, sendo observado pelos profissionais de saúde como capaz de proporcionar ao bebê benefícios durante o processo de realização desse método, auxiliando em resultados satisfatórios por meio da melhora da evolução clínica e sendo comprovado pelas falas a seguir:

\footnotetext{
"Enquanto o bebê está no Método Canguru, ele ganha peso mais rápido." (Francisca)

"No dia que é feito o Método Canguru, os nenéns têm uma tendência maior a ganhar peso." (Sheila)

"Vemos que o método nos ajuda no sentido de liberar essa criança mais rápido.” (Susi)

"Na prática, esse método vai beneficiar o bebê pelo resto de sua vida." (Kátia)
}

É notório, diante das falas, o benefício do método para a saúde dos neonatos. O contato entre mãe e bebê durante a utilização do método estimula o ganho de peso ponderal de forma mais acelerada, a partir do controle da termorregulação e da promoção do aleitamento materno ${ }^{(13)}$. A mãe que consegue enfrentar de forma ativa esse processo oferece ao filho prematuro um ambiente suficientemente bom para favorecer o seu crescimento ${ }^{(19)}$.

Outra maneira de o MMC contribuir para os resultados positivos com o RN prematuro é compreendida pelos profissionais de enfermagem ao manter o bem-estar físico do $\mathrm{RN}$ promovendo a redução na frequência respiratória e pressão arterial média, aumentando a saturação de oxigênio e normalizando a temperatura corporal ${ }^{(19,20)}$. Isso também foi observado na fala dos participantes, como é evidenciado a seguir:

\footnotetext{
"Vai ajudar manter a temperatura." (Susi)

"A gente percebe que o bebê fica mais calmo, para de chorar. Às vezes, até dorme." (Francisca)

"Acalma a criança." (Maria)

"Vai ter um bem estar enquanto o método é proporcionado." (Susi)
}

Outro estudo ${ }^{(21)}$ aponta que os resultados não mostraram alterações significativas quanto à pressão arterial média ( $p>0,05)$ e frequência cardíaca $(p>0,05)$ após a aplicação do MMC, porém, houve um aumento significativo da temperatura axilar $(p<0,05)$ e da saturação periférica de oxigênio $(p<0,05)$, e diminuição significativa da frequência respiratória $(\mathrm{p}<0,05)$, concluindo assim que esse método promove a melhora da temperatura corporal e o aumento da saturação periférica de oxigênio dos $\mathrm{RN}$ pré-termos. Além disso, autores ${ }^{(13)}$ informam que quando há amamentação precocemente com um bebê prematuro, há ganho de peso, aumento do nível de glicose no sangue e diminuição da bilirrubina não conjugada, auxiliando no desenvolvimento intelectual e neurológico.
Os profissionais de enfermagem identificaram, ainda, ao vivenciarem o fenômeno MMC como um cuidado humanizado ao $\mathrm{RN}$, que essa vivência os leva a prestar cuidados de enfermagem ao RN como um cuidado que faz parte da rotina do seu trabalho na maternidade, contribuindo para uma assistência de qualidade ao $\mathrm{RN}$, conforme os discursos a seguir:

"Na hora de fazer o método, é mais um cuidado que a gente tem que fazer." (Sheila)

"Mais um instrumento que a gente pode utilizar para tá melhorando a assistência." (Susi)

"Contribui para uma assistência mais humanizada." (Kátia)

"Método de cuidado de observação com o bebê aqui ou lá fora." (Maria)

É primordial que os profissionais de enfermagem assumam a postura de cuidador e acolhedor, otimizando o cuidado ao binômio mãe-filho ${ }^{(17)}$.

Portanto, a enfermagem exerce um papel indispensável na inserção da família ao método, sendo necessário conhecer, compreender e transformar sua assistência, vendo mãe, bebê e família dentro de suas culturas próprias, entendendo a necessidade de promover cuidados de enfermagem na perspectiva transcultural ${ }^{(14,15)}$. Tal inserção contribui para minimizar o impacto da estranheza causado pelo ambiente hospitalar, o qual é permeado por recursos tecnológicos que são poucos conhecidos pelos pais, e onde a comunicação dos profissionais com os pais é construída, muitas vezes, por uma linguagem constituída de termos técnicos ${ }^{(15)}$

Para que se possa manter essa assistência de qualidade, os profissionais de enfermagem vivenciam o MMC como uma oportunidade de promover a educação em saúde.

"Melhor explicado para a mãe para melhor adesão." (Kátia)

"Eu vou ensinar e observar elas daqui." (Maria)

"Eu vejo muito mais a mãe pedindo porque alguém orientou." (Susi)

"O cuidado que a gente ensina e orienta elas devem continuar em casa." (Maria)

Em estudo realizado em São Luís-MA, em 2005, com 30 mães, os benefícios do trabalho educativo realizado pela equipe de enfermagem com as mães foram confirmados na prática domiciliar, com $93,3 \%$ das mães adotando adequadamente a posição canguru, 86,7\% amamentando tecnicamente correto e sem fazer uso de qualquer tipo de adereços e $46,7 \%$ permanecendo de cinco a oito horas por dia com o bebê nessa posição ${ }^{(22)}$.

A enfermagem deve educar no decorrer da vivência materna no MMC, gerando uma assistência humanizada e educativa que será imprescindível para toda a vida 
familiar ${ }^{(14)}$, instruindo os pais a compreenderem esse processo antecipado do nascimento e preparando-os para o cuidado após a alta ${ }^{(13,23)}$. Em estudo conduzido com 12 trabalhadores de um hospital materno-infantil da cidade do Rio de Janeiro-RJ, os profissionais destacaram o chamado "cuidado ampliado", relacionado à preocupação da equipe em integrar participativamente os familiares que acompanham os RN no processo de internação ${ }^{(15)}$.

Além disso, neste estudo, foi percebida por alguns entrevistados a necessidade da educação continuada para aprimoramento profissional, o que é evidenciado nas falas a seguir.

"Precisa ser aprimorado aqui para nós, profissionais." (Susi)

"No ponto profissional, foi uma descoberta." (Sheila)

"Muita gente não sabe para que ou o que isso significa." (Susi)

Os profissionais conhecem o método empiricamente, aceitam-no e estão disponíveis para a sua implementação por meio do treinamento e composição de uma equipe multiprofissional sem papéis hierárquicos, com reconhecimento de ações compartilhadas para o trabalho integrado e sem dicotomias de informações para mãe e familiares, o que corrobora com afirmações de outros autores $^{(24)}$.

Em estudos ${ }^{(25,26)}$, verificou-se que, apesar do conhecimento teórico sobre a atenção humanizada ao RN por meio do $\mathrm{MMC}$, os profissionais ainda não o utilizam plenamente em sua prática clínica, sugerindo que ainda não está completamente assimilada a abrangência dessa forma de cuidado neonatal. Esses mesmos autores recomendam a educação permanente em todos os níveis de formação dos profissionais de saúde que prestam assistência ao RN. O sucesso do processo de mudança pressupõe a sensibilização e a educação permanente das pessoas envolvidas ${ }^{(18)}$.

\section{CONSIDERAÇÕES FINAIS}

A partir do estudo, foi possível perceber que os profissionais de enfermagem entrevistados compreendem o MMC aplicado na sua assistência diária como uma busca pela humanização do cuidado ao $\mathrm{RN}$, como uma forma de estímulo à ligação entre mãe e filho, e se apresenta como fator de relevância na recuperação do $\mathrm{RN}$, proporcionando aumento de peso, estabilidade dos dados vitais e estímulo à amamentação. Para os entrevistados, a aprendizagem para aquisição de segurança quanto ao cuidado com esses bebês, por meio da promoção da saúde e educação continuada dos profissionais de saúde, é essencial para ofertar esse cuidado humanizado na assistência neonatal, sendo essa educação em saúde primordial para uma assistência de qualidade.
Ressalta-se que, dentre as dificuldades encontradas na condução do estudo, deparou-se com o fato de os profissionais conhecerem quase que de forma empírica o Método Mãe Canguru, restringindo o seu uso como rotina do setor, mesmo conhecendo os benefícios que o método traz ao binômio mãe-filho, deixando evidente que essa forma de cuidado ainda não está completamente assimilada por todos.

Recomenda-se a realização de outros estudos sobre essa temática, principalmente em outras unidades que prestam assistência neonatal, para uma melhor visibilidade da realização desse método, alicerçando a melhoria da qualidade da assistência à saúde do $\mathrm{RN}$ em relação ao MMC.

Sugere-se a continuidade das ações de assistência voltadas para a realização desse método pela equipe de saúde, contribuindo para a diminuição da morbimortalidade nessa clientela.

Por fim, destaca-se o caráter importante do presente estudo, que poderá servir como ponto de referência para outras pesquisas e/ou estudos a serem desenvolvidos, fortalecendo a linha de pesquisa sobre políticas na saúde do RN, favorecendo o aprimoramento de ações assistenciais que promovam o fortalecimento do $\mathrm{MMC}$, pois a equipe de saúde, além de estar motivada para implementar essa assistência, deve orientar e estimular as famílias a desenvolverem esse tipo de cuidado. É, pois, primordial, desenvolver espaços que propiciem reflexões para que esses profissionais possam discutir sobre sua prática diária e os reflexos dela na aplicação, desenvolvimento e avaliação do Método Mãe-Canguru.

\section{REFERÊNCIAS}

1. Silva JR, Thomé CR, Abreu RM. Método mãe canguru nos hospitais/maternidades públicos de Salvador e atuação dos profissionais da saúde na segunda etapa do método. Rev CEFAC. 2011;13(3):522-33.

2. Gontijo TL, Meireles AL, Malta DC, Proietti FA, Xavier CC. Evaluation of implementation of humanized care to low weight newborns - the Kanga-roo Method. J Pediatr. 2010; 86(1):33-9.

3. Ministério da Saúde (BR). Norma de orientação para implantação do projeto canguru. Brasília; 2000. n. 693.

4. Almeida H, Venancio SI, Sanches MTC, Onuki D. Impacto do método canguru nas taxas de aleitamento materno exclusivo em recém-nascidos de baixo peso. J Pediatr. 2010;86(3):250-3.

5. Blanca Gutiérrez JJ, Ábalos Pérez MR, Montes Aguilera MV, González Moreno S. The role of fathers 
in the postpartum period: experiences with skin to skin method. Acta Paul Enferm. 2012;25(6):914-20.

6. Matijasevich A. Estimativas corrigidas da prevalência de nascimentos pré-termo no Brasil, 2000 a 2011. Epidemiol Serv Saúde. 2013;22(4):557-64.

7. Davim RMB, Enders BC, Silva RAR. Mothers'feelings about breastfeeding their premature babies in a roominginfacility. Rev Esc Enferm USP. 2010;44(3):713-8.

8. Olmedo MD, Gabas GS, Merey LSF, Souza LS, Muller KTC, Santos MLM, et al. Respostas fisiológicas de recém-nascidos pré-termo submetidos ao Metódo Mãe-Canguru e a posição prona. Fisioter Pesqui. 2012;19(2):115-21.

9. Arivabene JC, Tyrrell MAR. Método mãe canguru: vivências maternas e contribuições para a enfermagem. Rev Latinoam Enferm. 2010;18(2):262-8.

10. Minayo MCS. O desafio do conhecimento: pesquisa qualitativa em saúde. São Paulo: HUCITEC; 2006.

11. Leopardi MT. Metodologia da pesquisa qualitativa. Santa Maria: Palotti; 2001.

12. Bardin L. Análise de conteúdo. Lisboa: Edições 70; 2009.

13. Casati PS, Oliveira CS, Paula S. Método Mãe Canguru e suas Associações nos Benefícios dos Recém-Nascidos Baixo Peso. UNICiências. 2010;14(1):135-46.

14. Neves PN, Ravelli APX, Lemos JRD. Atenção humanizada ao recém-nascido de baixo-peso (método mãe canguru): percepções de puérperas. Rev Gaúcha Enferm. 2010; 31(1):48-54.

15. Souza KMO, Ferreira SD. Assistência humanizada em UTI neonatal: os sentidos e as limitações identificadas pelos profissionais de saúde. Ciênc Saúde Coletiva. 2010;15(2):471-80.

16. Freitas JO, Camargo CL. Método Mãe-Canguru: evolução ponderal de recém-nascidos. Acta Paul Enferm. 2007;20(1):75-81.

17. Parisi TCH, Coelho ERB, Melleiro MM. Implantação do Método Mãe-Canguru na percepção de enfermeiras de um hospital universitário. Acta Paul Enferm. 2008;21(4):575-80.
18. Chagas DO, Meirilene Pereira AS, Nicomedes TM, Lima RABC, Azevedo VMGO, Gontijo FO. Comparação da adesão materna às orientações do método Mãe Canguru no pré e pós-alta do Hospital Sofia Feldman. Rev Méd Minas Gerais. 2011; 21(1):5-8

19. Sá FE, Costa FS, Pereira MLD, Dantas MA, Feitosa HN, Eleutério FJC. Sentimentos e emoções maternas na vivência do método mãe-canguru. Femina. 2006;34(2):135-40.

20. Moreira JO, Romagnoli RC, Dias DAS, Moreira CB. Programa mãe-canguru e a relação mãe-bebê: pesquisa qualitativa na rede pública de Betim. Psicol Estud. 2009; 14(3):475-83.

21. Almeida CM, Almeida AFN, Forti EMP. Efeitos do Método Mãe Canguru nos sinais vitais de recémnascidos pré-termo de baixo peso. Rev Bras Fisioter. 2007;11(1):1-5.

22. Araújo CL, Rios CTF, Santos MH, Gonçalves APF. Método Mãe Canguru: uma investigação da prática domiciliar. Ciênc Saúde Coletiva. 2010;15(1):301-7.

23. Cabral IE, Groleau D. A prática da amamentação após o método mãe canguru no Rio de Janeiro: a necessidade de educação em saúde e intervenção de Enfermagem no domicílio. Esc Anna Nery. 2009;13(4):763-71.

24. Sá FE, Sá RC, Pinheiro LMF, Callou FEO. Relações interpessoais entre os profissionais e as mães de prematuros da unidade canguru. Rev Bras Promoç Saúde. 2010;23(2):144-9.

25. Lazzari DD, Schmidt N, Jung W. Educação continuada em unidade de terapia intensiva na percepção de Enfermeiras. Rev Enferm UFSM. 2012;2(1):88-96.

26. Hennig MAS, Gomes MASM, Gianini NOM. Conhecimentos e práticas dos profissionais de saúde sobre a "atenção humanizada ao recém-nascido de baixo peso - método canguru”. Rev Bras Saude Mater Infant. 2006; 6(4):427-35.

\section{Endereço para correspondência:}

Luís Paulo Souza e Souza

Rua Doze, 47

Bairro: Santo Antônio

CEP: 39402-285 - Montes Claros - MG - Brasil

E-mail: luis.pauloss@hotmail.com 